\title{
A note on the validity of the Schrödinger approximation for the Helmholtz equation
}

\author{
Maximilian Klumpp, Guido Schneider \\ Institut für Analysis, Dynamik und Modellierung, \\ Universität Stuttgart, Pfaffenwaldring 57, \\ 70569 Stuttgart, Germany
}

April 2, 2021

\begin{abstract}
Time-harmonic electromagnetic waves in vacuum are described by the Helmholtz equation $\Delta u+\omega^{2} u=0$ for $(x, y, z) \in \mathbb{R}^{3}$. For the evolution of such waves along the $z$-axis a Schrödinger equation can be derived through a multiple scaling ansatz. It is the purpose of this paper to justify this formal approximation by proving bounds between this formal approximation and true solutions of the original system. The challenge of the presented validity analysis is the fact that the Helmholtz equation is ill-posed as an evolutionary system along the $z$-axis.
\end{abstract}

\section{Introduction}

Electromagnetic waves are described by the system of Maxwell's equations [3]. In vacuum this system of equations reduce to the scalar linear wave equation

$$
\partial_{t}^{2} u=\Delta u=\partial_{x}^{2} u+\partial_{y}^{2} u+\partial_{z}^{2} u,
$$

with $u(x, y, z, t), x, y, z, t \in \mathbb{R}$. For time harmonic waves

$$
u(x, y, z, t)=v(x, y, z) e^{i \omega t}
$$


we find

$$
-\omega^{2} v=\partial_{x}^{2} v+\partial_{y}^{2} v+\partial_{z}^{2} v
$$

We are interested in the evolution of waves along the z-axis and so we write (1) as evolutionary system w.r.t. the $z$-variable. The resulting system

$$
\partial_{z}^{2} v=-\omega^{2} v-\partial_{x}^{2} v-\partial_{y}^{2} v
$$

is solved by harmonic waves

$$
v(x, y, z)=e^{\lambda z+i k_{x} x+i k_{y} y},
$$

with $\lambda\left(k_{x}, k_{y}\right), k_{x}, k_{y} \in \mathbb{R}$ and

$$
\lambda^{2}=k_{x}^{2}+k_{y}^{2}-\omega^{2}
$$

Since (1) is an elliptic system the z-evolutionary system (2) in general is an ill-posed initial value problem. Out of this ill-posed system a well-posed initial value problem can be derived through some multiple scaling analysis. In detail, inserting the ansatz

$$
v(x, y, z) \approx \psi_{\text {app }}(x, y, z)=e^{i k_{z} z} w(X, Y, Z)
$$

with

$$
X=\varepsilon x, \quad Y=\varepsilon y, \quad Z=\varepsilon^{2} z,
$$

and $0<\varepsilon \ll 1$ a small perturbation parameter, yields

$$
-k_{z}^{2} w+2 i \varepsilon^{2} k_{z} \partial_{Z} w+\varepsilon^{4} \partial_{Z}^{2} w=-\omega^{2} w-\varepsilon^{2} \partial_{X}^{2} w-\varepsilon^{2} \partial_{Y}^{2} w
$$

see the subsequent Remark 2.4 for an additional discussion. Choosing $\omega^{2}=$ $k_{z}^{2}$ and ignoring the terms of order $\mathcal{O}\left(\varepsilon^{4}\right)$ gives that $w$ has to satisfy in lowest order the Schrödinger equation

$$
2 i k_{z} \partial_{Z} w=-\partial_{X}^{2} w-\partial_{Y}^{2} w
$$

which, in contrast to the Helmholtz equation (2), is a well-posed initial value problem w.r.t. the evolutionary variable $Z$. This formal approach is widely used in the physics and engineering literature for instance to describe the evolution of light beams in vacuum and media [8, 1], to compute beam quality factors in step-index fibers [11, or in the modeling of physical optics 
phenomena by complex ray tracing [2]. It is known as the "paraxial approximation". A motivation of this approximation is given in [6], but, to our knowledge, no rigorous analysis has been made so far to justify this formal approximation. Therefore, it is the purpose of this note to prove rigorous bounds between this formal approximation and true solutions of the original system for small $\varepsilon>0$. Such estimates are known for dispersive/hyperbolic systems already for many years, see Remark 2.3.

\section{The approximation result}

Notation. Many possibly different constants are denoted with the symbol $C$ if they can be chosen independently of the small perturbation parameter $0<\varepsilon \ll 1$. The Fourier transform of a function $u$ w.r.t. the variables $x$ and $y$ is denoted with $\widehat{u}$ and is given by

$$
\widehat{u}\left(k_{x}, k_{y}\right)=\frac{1}{(2 \pi)^{2}} \int_{\mathbb{R}^{2}} u(x, y) e^{-i k_{x} x-i k_{y} y} d(x, y) .
$$

The Sobolev space $H^{s}$ is equipped with the norm

$$
\|u\|_{H^{s}}=\sum_{m_{1}+m_{2} \leq s, m_{1}, m_{2} \in \mathbb{N}_{0}}\left(\int_{\mathbb{R}^{2}}\left|\partial_{x}^{m_{1}} \partial_{y}^{m_{2}} u\right|^{2} d(x, y)\right)^{1 / 2}
$$

We introduce the weighted Lebesgue space $L_{s}^{2}$ equipped with the norm

$$
\|\widehat{u}\|_{L_{s}^{2}}=\left(\int_{\mathbb{R}^{2}}\left|\widehat{u}\left(k_{x}, k_{y}\right)\right|^{2} \rho(|k|)^{2 s} d\left(k_{x}, k_{y}\right)\right)^{1 / 2}
$$

where $\rho(|k|)=\left(1+|k|^{2}\right)^{1 / 2}$ and $|k|^{2}=k_{x}^{2}+k_{y}^{2}$. We use that Fourier transform is an isomorphism between $H^{s}$ and $L_{s}^{2}$, i.e., for $s \in \mathbb{N}_{0}$ there exist $C_{1}, C_{2}>0$ such that for all $u \in H^{s}$

$$
\|\widehat{u}\|_{L_{s}^{2}} \leq C_{1}\|u\|_{H^{s}} \leq C_{2}\|\widehat{u}\|_{L_{s}^{2}} .
$$

See [10, Lemma 7:3.31]. Hence, for all $s \geq 0$ we redefine $\|u\|_{H^{s}}=\|\widehat{u}\|_{L_{s}^{2}}$.

Then we prove the following approximation result. 
Theorem 2.1. Fix $s_{A} \geq \max (4, s)$ and $Z_{0}>0$. Let $w \in C\left(\left[0, Z_{0}\right], H^{s_{A}}\right)$ be a solution of the Schrödinger equation (5). Then there exist $a C>0$ and an $\varepsilon_{0}>0$ such that for all $\varepsilon \in\left(0, \varepsilon_{0}\right)$ there are solutions $v$ of (2) with

$$
\sup _{z \in\left[0, Z_{0} / \varepsilon^{2}\right]}\left\|v(x, y, z)-e^{i k_{z} z} w\left(\varepsilon x, \varepsilon y, \varepsilon^{2} z\right)\right\|_{H^{s}(d x, d y)} \leq C \varepsilon,
$$

In particular, by Sobolev's embedding theorem we have

$$
\sup _{z \in\left[0, Z_{0} / \varepsilon^{2}\right]} \sup _{x, y \in \mathbb{R}^{2}}\left|v(x, y, z)-e^{i k_{z} z} w\left(\varepsilon x, \varepsilon y, \varepsilon^{2} z\right)\right| \leq C \varepsilon .
$$

Remark 2.2. The challenge of the presented validity analysis is the fact that in every $H^{s}$ the Helmholtz equation is ill-posed as an evolutionary system along the $z$-axis.

Remark 2.3. The method presented in this note does not apply to nonlinear problems since it uses a cut-off in Fourier space which is not respected by nonlinear terms. Hence, it cannot be used to justify the NLS equation for weakly nonlinear elliptic Maxwell models. See for instance [4, 5, 9] for validity results for the NLS approximation of nonlinear dispersive/hyperbolic systems and the textbooks [7, 10] for an introduction and a recent overview.

Remark 2.4. Alternatively to the scaling (44) we can introduce the small parameter $0<\varepsilon \ll 1$ already at the beginning by considering

$$
u(x, y, z, t)=v(x, y, z) e^{i \omega t / \varepsilon^{2}}
$$

which can be physically motivated by the highly oscillatory character of light. The ansatz is then given by

$$
\psi_{\text {app }}(x, y, z)=e^{i k_{z} z / \varepsilon^{2}} w(x, y, z),
$$

which yields as above the Schrödinger equation (5). It is an easy exercise to reformulate Theorem 2.1 and the subsequent proof w.r.t. this scaling.

\section{The proof}

We have that for fixed $\omega$ the eigenvalues $\lambda$ defined by (3) are purely imaginary for $k_{x}^{2}+k_{y}^{2} \leq \omega^{2}$ and real-valued for $k_{x}^{2}+k_{y}^{2} \geq \omega^{2}$ with $\left|\lambda\left(k_{x}, k_{y}\right)\right| \rightarrow \infty$ for $k_{x}^{2}+k_{y}^{2} \rightarrow \infty$. Since (2) is then an ill-posed initial value problem we use a 
cut-off function in Fourier space to remove the ill-posed part of (2). In detail, we define a projection $P_{h y p}$ on the hyperbolic part of (2) by

$$
P_{\text {hyp }} u=\mathcal{F}^{-1} \chi \mathcal{F} u
$$

where

$$
\chi(k)= \begin{cases}1, & \text { for } k_{x}^{2}+k_{y}^{2} \leq \omega^{2} / 2, \\ 0, & \text { else. }\end{cases}
$$

Moreover, let $P_{\text {ell }}=I-P_{\text {hyp }}$. For all $s \geq 0$ we have

$$
\left\|P_{h y p} u\right\|_{H^{s}} \leq\|\chi \widehat{u}\|_{L_{s}^{2}} \leq\|\widehat{u}\|_{L_{s}^{2}} \leq\|u\|_{H^{s}}
$$

and similarly $\left\|P_{\text {ell }} u\right\|_{H^{s}} \leq\|u\|_{H^{s}}$. For the subsequent estimates it turns out to be advantageous to take $\omega^{2} / 2$ instead of $\omega^{2}$ in the definition of $P_{\text {hyp }}$. In order to estimate the difference between solutions $v$ of (2) and the Schrödinger approximation $\psi_{\text {app }}$ we work with the hyperbolic part $P_{h y p} \psi_{a p p}$ of the Schrödinger approximation. Then we use the triangle inequality to estimate

$$
\left\|v-\psi_{a p p}\right\|_{H^{s}} \leq\left\|v-P_{h y p} \psi_{a p p}\right\|_{H^{s}}+\left\|P_{h y p} \psi_{\text {app }}-\psi_{a p p}\right\|_{H^{s}} .
$$

The two terms on the right hand side are estimated in the following two subsections.

\subsection{Estimating the well-posed part}

We consider (2) with initial conditions $\left.P_{h y p} \psi_{a p p}\right|_{z=0}$ and $\left.\partial_{z} P_{h y p} \psi_{a p p}\right|_{z=0}$. Since (2) is a linear system, the Fourier support of the solution $v$ of (2) is preserved. The difference $R=v-P_{\text {hyp }} \psi_{\text {app }}$ then satisfies

$$
\partial_{z}^{2} R=-\partial_{x}^{2} R-\partial_{y}^{2} R-\omega^{2} R-\varepsilon^{4} e^{i k_{z} z} P_{h y p} \partial_{Z}^{2} w,
$$

with initial data $\left.R\right|_{z=0}=0$ and $\left.\partial_{z} R\right|_{z=0}=0$. The inhomogeneity can be expressed via the right hand side of the Schrödinger equation, namely

$$
\partial_{Z}^{2} w=-\partial_{Z}\left(\frac{1}{2 i k_{z}}\left(\partial_{X}^{2} w+\partial_{Y}^{2} w\right)\right)=-\frac{1}{4 k_{z}^{2}}\left(\partial_{X}^{2}+\partial_{Y}^{2}\right)^{2} w
$$

For $w \in C\left(\left[0, Z_{0}\right], H^{s_{A}}\right)$ we thus have $\partial_{Z}^{2} w \in C\left(\left[0, Z_{0}\right], H^{s_{A}-4}\right)$, i.e., there exist $C_{1}, C_{\text {res }}>0$ such that

$$
\left\|\varepsilon^{4} P_{h y p} \partial_{Z}^{2} w\right\|_{L^{2}} \leq C_{1} \varepsilon^{4}\left\|\left(\partial_{X}^{2}+\partial_{Y}^{2}\right)^{2} w\right\|_{L^{2}} \leq C_{r e s} \varepsilon^{4}\|w\|_{H^{4}} \leq C_{r e s} \varepsilon^{4}\|w\|_{H^{s} A}
$$


for all $z \geq 0$. Since for functions with compact Fourier support each $H^{s}$-norm can be estimated by the $L^{2}$-norm, in particular for $u$ with $u=P_{h y p} u$ we have

$$
\|u\|_{H^{s}}=\|\chi \widehat{u}\|_{L_{s}^{2}} \leq\left\|\chi \widehat{u} \rho^{s}\right\|_{L_{0}^{2}} \leq\left\|\chi \rho^{s}\right\|_{L^{\infty}}\|\widehat{u}\|_{L_{0}^{2}} \leq C\|u\|_{L^{2}},
$$

with a constant $C_{s}$ for each fixed $s$. Therefore, we finally have

$$
\left\|\varepsilon^{4} P_{h y p} \partial_{Z}^{2} w\right\|_{H^{s}} \leq C_{s}\left\|\varepsilon^{4} P_{h y p} \partial_{Z}^{2} w\right\|_{L^{2}} \leq C_{s} C_{r e s} \varepsilon^{4}\|w\|_{H^{s_{A}}} .
$$

In Fourier space, w.r.t. $x$ and $y$, (8) is given by

$$
\partial_{z}^{2} \widehat{R}=-\widehat{\omega}^{2} \widehat{R}-\varepsilon^{4} e^{i k_{z} z} \chi \partial_{Z}^{2} \widehat{w}
$$

with $\widehat{\omega}^{2}\left(k_{x}, k_{y}\right)=\omega^{2}-k_{x}^{2}-k_{y}^{2}$ and $\widehat{R}, \widehat{w}$ is the Fourier transform w.r.t. the variables $X, Y$. Multiplying (10) with $\overline{\partial_{z} \widehat{R}}$ and integrating w.r.t. $k_{z}$ and $k_{y}$ yields

$$
\begin{aligned}
\frac{d}{d z} \int_{\mathbb{R}^{2}}\left|\partial_{z} \widehat{R}\right|^{2}+|\widehat{\omega} \widehat{R}|^{2} d\left(k_{x}, k_{y}\right) & \leq 2\left|\operatorname{Re} \int_{\mathbb{R}^{2}} \overline{\partial_{z} \widehat{R}}\left(\varepsilon^{4} \chi \partial_{Z}^{2} \widehat{w}\right) d\left(k_{x}, k_{y}\right)\right| \\
& \leq \varepsilon^{2} \int_{\mathbb{R}^{2}}\left|\partial_{z} \widehat{R}\right|^{2} d\left(k_{x}, k_{y}\right)+\varepsilon^{6} \int_{\mathbb{R}^{2}}\left|\chi \partial_{Z}^{2} \widehat{w}\right|^{2} d\left(k_{x}, k_{y}\right) .
\end{aligned}
$$

The second summand can be estimated by

$$
\begin{aligned}
\varepsilon^{6} \int_{\mathbb{R}^{2}}\left|\chi \partial_{Z}^{2} \widehat{w}\right|^{2} d\left(k_{x}, k_{y}\right) & =\varepsilon^{6}\left\|\chi \partial_{Z}^{2} \widehat{w}\right\|_{L^{2}\left(d k_{x}, d k_{y}\right)}^{2}=\varepsilon^{6}\left\|P_{h y p} \partial_{z}^{2} w\right\|_{L^{2}(d x, d y)}^{2} \\
& \leq \varepsilon^{4}\left\|P_{h y p} \partial_{z}^{2} w\right\|_{L^{2}(d X, d Y)}^{2} \leq C_{r e s} \varepsilon^{4}\|w\|_{H^{s}(d X, d Y)} .
\end{aligned}
$$

Thus, for $E=\int\left|\partial_{z} \widehat{R}\right|^{2}+|\widehat{\omega} \widehat{R}|^{2} d\left(k_{x}, k_{y}\right)$ we find

$$
\frac{d}{d z} E \leq \varepsilon^{2} E+\varepsilon^{4} C_{R e s}^{2}\|w\|_{C\left(\left[0, Z_{0}\right], H^{4}\right)}^{2} .
$$

Gronwall's inequality then yields

$$
E(z) \leq \int_{0}^{z} e^{\varepsilon^{2}(z-s)} \varepsilon^{4} C_{R e s}^{2}\|w\|_{C\left(\left[0, Z_{0}\right], H^{4}\right)}^{2} d s \leq C \varepsilon^{2}\|w\|_{C\left(\left[0, Z_{0}\right], H^{4}\right)}^{2}
$$

for $z \in\left[0, Z_{0} / \varepsilon^{2}\right]$. Since by construction $\widehat{\omega} \chi$ is bounded away from zero, $E(z)^{1 / 2}$ is an upper bound for the $L^{2}$-norm of $\widehat{R}$ and thus by Parseval's inequality an upper bound for the $L^{2}$-norm of $R$, in detail

$$
\|R\|_{L^{2}}=\|\widehat{R}\|_{L^{2}} \leq C_{3}\|\widehat{\omega} \widehat{R}\|_{L^{2}} \leq C_{3} E(z)^{1 / 2}
$$

for some $C_{3}>0$. Since $R$ has a compact support in Fourier space, as in (9) the $L^{2}$-estimate implies an $H^{s}$-estimate for $R$ for every $s \geq 0$. 


\subsection{Estimating the ill-posed part}

For estimating the term $\left\|P_{h y p} \psi_{a p p}-\psi_{a p p}\right\|_{H^{s}}$ we use that

$$
\widehat{\psi}_{\text {app }}\left(k_{x}, k_{y}, z\right)=\varepsilon^{-2} \widehat{w}\left(\frac{k_{x}}{\varepsilon}, \frac{k_{y}}{\varepsilon}, \varepsilon^{2} z\right) e^{i k_{z} z}
$$

is strongly concentrated at the wave vector $\left(k_{x}, k_{y}\right)=(0,0)$. For $s_{A} \geq s$ we estimate

$$
\begin{aligned}
& \left\|P_{h y p} \psi_{\text {app }}-\psi_{\text {app }}\right\|_{H^{s}} \\
& \stackrel{\underline{6}}{\leq} C\left\|\widehat{P}_{h y p} \widehat{\psi}_{\text {app }}-\widehat{\psi}_{\text {app }}\right\|_{L_{s}^{2}} \stackrel{\underline{\underline{7}}}{=} C\left\|(1-\chi) \widehat{\psi}_{\text {app }}\right\|_{L_{s}^{2}} \\
& =C\left\|(1-\chi(k)) \varepsilon^{-2} \widehat{w}\left(\frac{k_{x}}{\varepsilon}, \frac{k_{y}}{\varepsilon}, \varepsilon^{2} z\right) \rho(k)^{s}\right\|_{L^{2}\left(d k_{x}, d k_{y}\right)} \\
& \leq C \sup _{k \in \mathbb{R}^{2}}\left|(1-\chi(k)) \frac{\rho(k)^{s}}{\rho\left(\frac{k}{\varepsilon}\right)^{s_{A}}}\right|\left\|\varepsilon^{-2} \widehat{w}\left(\frac{k_{x}}{\varepsilon}, \frac{k_{y}}{\varepsilon}, \varepsilon^{2} z\right) \rho\left(\frac{k}{\varepsilon}\right)^{s_{A}}\right\|_{L^{2}\left(d k_{x}, d k_{y}\right)} \\
& \leq C \varepsilon^{s_{A}-1}\left\|\widehat{w}\left(K_{x}, K_{y}\right)\right\|_{L_{s_{A}}^{2}\left(d K_{x}, d K_{y}\right)} \stackrel{\sqrt[6]{\leq}}{\leq} C \varepsilon^{s_{A}-1}\|w\|_{H^{s_{A}}},
\end{aligned}
$$

with $K_{x}=\frac{k_{x}}{\varepsilon}, K_{y}=\frac{k_{y}}{\varepsilon}$, where the loss of $\varepsilon^{-1}$ comes from the scaling properties of the $L^{2}$-norm and of Fourier transform.

\subsection{Summary}

Combining the estimates of Section 3.1 and Section 3.2 gives via the triangle inequality

$$
\begin{aligned}
\left\|v-\psi_{a p p}\right\|_{H^{s}} & \leq\left\|v-P_{h y p} \psi_{a p p}\right\|_{H^{s}}+\left\|P_{h y p} \psi_{a p p}-\psi_{a p p}\right\|_{H^{s}} \\
& \leq C_{3} \sqrt{C \varepsilon^{2}}\|w\|_{C\left(\left[0, Z_{0}\right], H^{4}\right)}+C \varepsilon^{s_{A}-1}\|w\|_{C\left(\left[0, Z_{0}\right], H^{s} A\right)} \\
& \leq \tilde{C} \varepsilon
\end{aligned}
$$

for $z \in\left[0, Z_{0} / \varepsilon^{2}\right]$ and $s_{A} \geq \max (4, s)$. Therefore, we are done.

\section{References}

[1] D.Rafferty, U.H. Wagner, C. Rau, P. Chang, S. Alcock, R. Dockree, and I.R. Robinson. Development of a computer model to simulate wavefront propagation. 
[2] James E. Harvey, Ryan G. Irvin, and Richard N. Pfisterer. Modeling physical optics phenomena by complex ray tracing. Optical Engineering, 54(3):035105, 2015.

[3] John David Jackson. Classical Electrodynamics. Wiley, New York, 1988.

[4] L. A. Kalyakin. Asymptotic decay of a one-dimensional wave-packet in a nonlinear dispersive medium. Math. USSR, Sb., 60(2):457-483, 1988.

[5] Pius Kirrmann, Guido Schneider, and Alexander Mielke. The validity of modulation equations for extended systems with cubic nonlinearities. Proc. R. Soc. Edinb., Sect. A, Math., 122(1-2):85-91, 1992.

[6] M. Marte and S. Stenholm. Paraxial light and atom optics: The optical schrödinger equation and beyond. Physical Review A, 56:2940-2953, 1997.

[7] Jeffrey Rauch. Hyperbolic partial differential equations and geometric optics., volume 133. Providence, RI: American Mathematical Society (AMS), 2012.

[8] Jason Daniel Schmidt. Numerical simulation of optical wave propagation with examples in MATLAB. Bellingham, Wash. : SPIE monograph, PM199, 2010.

[9] Guido Schneider. Justification and failure of the nonlinear Schrödinger equation in case of non-trivial quadratic resonances. J. Differ. Equations, 216(2):354-386, 2005.

[10] Guido Schneider and Hannes Uecker. Nonlinear PDEs. A dynamical systems approach., volume 182. Providence, RI: American Mathematical Society (AMS), 2017.

[11] Hidehiko Yoda, Pavel Polynkin, and Masud Mansuripur. Beam quality factor of higher order modes in a step-index fiber. J. Lightwave Technol., 24(3):1350, Mar 2006. 